\title{
دور المشاريع العمرانية في تعزيز السياحة الحضرية

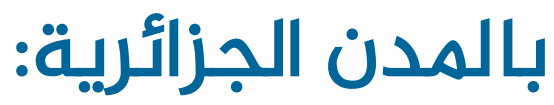 \\ فرص، تحولات وتحديات، حالة: مدينة سطيف
}

\section{فؤاد بن غضبان}

مخبر تقييم جودة الاستخدام في العمارة والبيئة المبنية (LEQUAE)

معهد تسيير التقنيات الحضرية- جامعة أم البواقي- الجزائر

foued.benghadbane@univ-oeb.dz

\section{محفوظ جعجو}

قسم الهندسة المعمارية- جامعة الشلف- الجزائر

mahfouddj89@gmail.com 

www.refaad.com
International Journal of Hospitality and Tourism Studies

(IJHTS)

Journal Homepage: https://www.refaad.com/views/IJHTS/home.aspx

ISSN 2709-0892 (Online) ISSN 2709-0884 (Print)

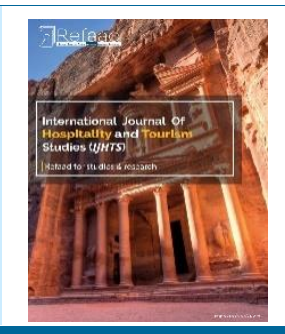

\title{
دور المشـاريع العمر انية في تعزيز السياحة الحضيرية بالمدن الجز ائرية: فرص، تحولات وتحديات، حالة: مدينة سطيف
}

\author{
فؤاد بن غضببان \\ مخبر تقييم جودة الاستخدام في العمارة والبيئة المبنية (LEQUAE) \\ معهد تسيير التقنيات الحضرية- جامعة أم البواقي- الجزائر \\ foued.benghadbane@univ-oeb.dz
}

\author{
محفوظ جعجو \\ قسم الهندسة المعمارية- جامعة الشلف- الجزائر \\ mahfouddj89@gmail.com
}

DOI: https://doi.org/10.31559/IJHTS2021.2.1.1 2021/6/21 2021 مراجتلام البحث: 2021/5/18 قبول البحث

لمر يعلد مفهوم السياحة يقتصر على السفر بهلف رؤية أماكن أخرى متميزة بثقافات وعادات مختلفة فقط، بل اتسع لتكون السياحة أكثر

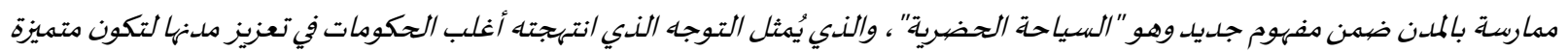

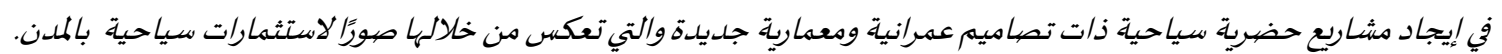

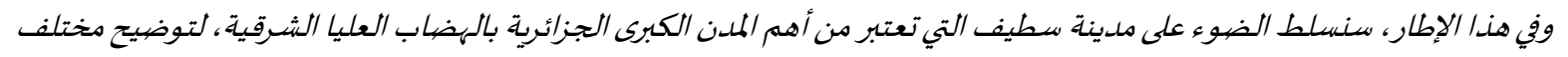
التحولات الحضرية والوظيفية الهامة الناتجة عن توطن العديد من المشاريع العمدانية (سلسلة الفنادق، المساكز التجارية الكبوى، الترامواي...)، وكذا إعادة تنظيم مختلف الوظائف الحضرية بها، والتي سمحت بتوفير العديد من مناطق التوفيه والاستجمام وتهيئتها،

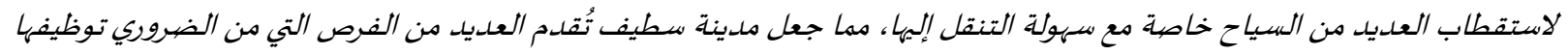
لتطوير السياحة الحضرية بها.

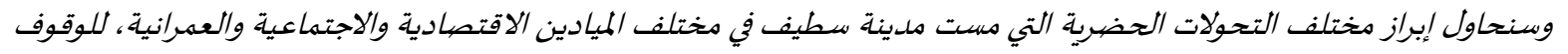
على كل المشاكل المعيقة لتنمية السياحة الحضرية بها بالاعتماد على المنهجين الوصفي والكمي وبعض التقنيات التكتيكية وكذا مجموعة

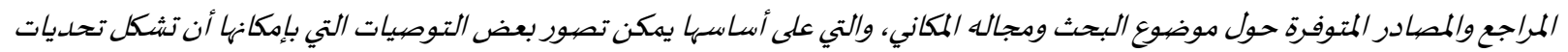

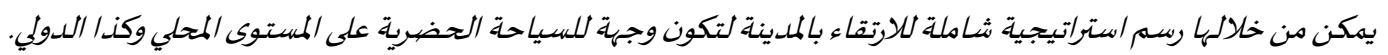
الكلمات المفتاحية: سياحة حضرية؛ فرص؛ تحولات؛ مناطق التوفيا والاستجمام؛ تحديات؛ مدينة سطيف؛ الجزائر.

يهدف التخطيط الحضري إلى توفير مجال حضري متوازن ومتلائم مع احتياجات سكانه، الذي يمارسون فياه نشاطاتهم اليومية ووفق منطق يقوم على المشاركة المجتمعية وتحقيق دور الفاعل الحقيقي في تخطيط المجال الحضري، ويتجسد هذا الدور ميدانيًا من خلال توطين مجموعة من

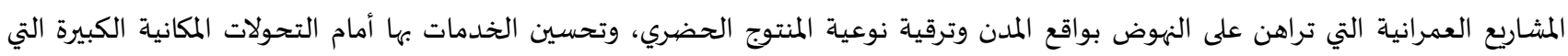
تشهدها المدينة اليوم في شتى المجالات الاقتصادية، الاجتماعية، السياسية...، ومن هنا تبرز أهمية المشاريع العمرانية في توفير مجال حضري يجمئ ولمع كل 
الشروط اللازمة لحياة السكان وتحسين مستواهم الاجتماعي من خلال تقديم الخدمات الحضرية في جميع الميادين والمجالات كالنقل، وتوفير فضاءات الترفياء وقضاء أوقات الفراغ، وإيجاد مناطق للتسوق،... وغيرها (Yildiz, Akbulut, 2013)، وتندرج كل هذه العمليات التخطيطية من منظور السياحة ضمن مفهوم التخطيط السياحي. (بن غضببان، 2019)

وقد ساهمت التحولات الاقتصادية والاجتماعية بالمدن خلال السنوات الأخيرة الماضية إلى إعادة تركيب بنيتها الحضرية استجابة لتوجهها

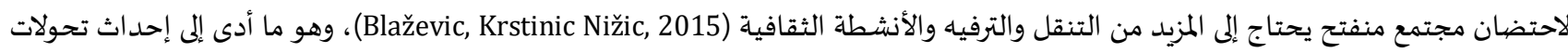

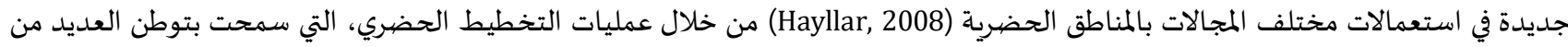

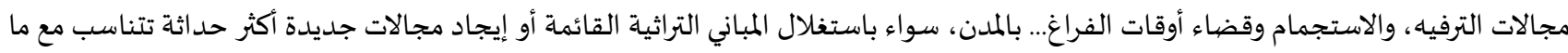
أوجدتا ثورة تكنولوجيا المعلومات والاتصالات، مما جعل مفهوم السياحة يفقد معناه التقليدي المتمحور حول السفر بهدف رؤية أماكن أخرى متميزة

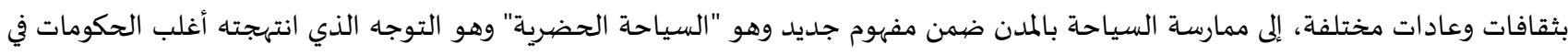

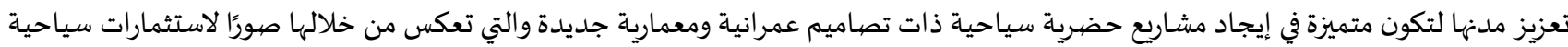

جديدة بالمدن. (Yildiz, Akbulut, 2013)

1.1. مشكلة الدراسـة:

أصبتحت المدن خاصة الكبرى أهم الوجهات السياحية في العالم، فهي تستقبل السياح المحليين والدوليين، وتعتبر السياحة الحضرية بمثابة

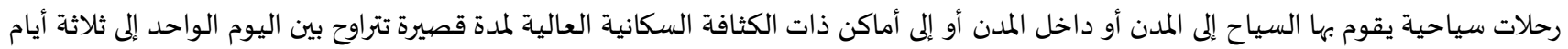

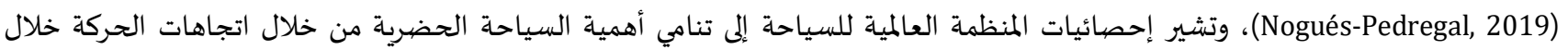
العطل؛ حيث ارتفعت نسبة الرحلات السياحية للمدينة بنسبة 21\% خلال السنوات الخمس الماضية (Bock, 2015).

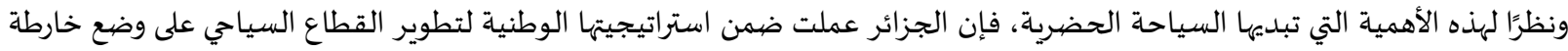

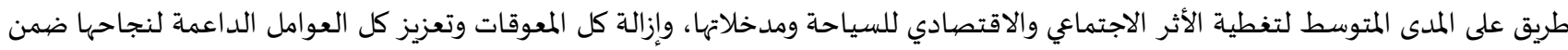

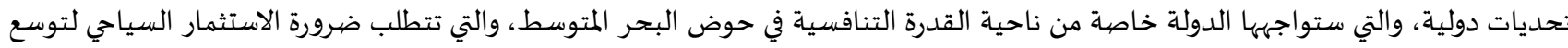

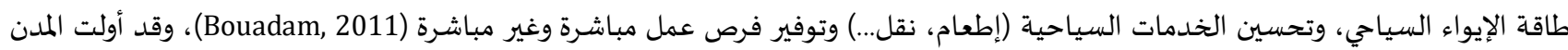

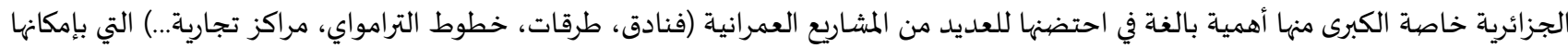
النهوض بالسياحة الحضرية. وفي ذات الوقت، فإن المدن الجزائرية باختلاف مواقعها الجغرافية (ساحلية، جبلية، صحراوية...) تتوفر على العديد من المقومات العمرانية

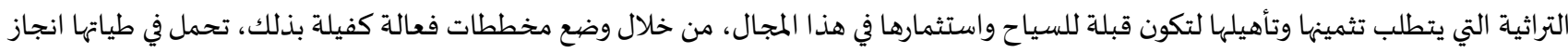
العديد من عمليات التخطيط الحضري التي تعمل على تسهيل الوصولية إليها وتجهيزها بالخدمات الضرورية لتلبية احتياجات المترددين عليها. (بن

غضببان، 2015)

وفي هذا الإطار، تبرز مدينة سطيف كمدينة جزائرية كبيرة قطعت خطوة كبيرة في مجال الاستثمار السياحي من خلال ترقية وتطوير وتنويع

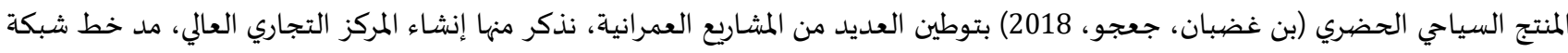

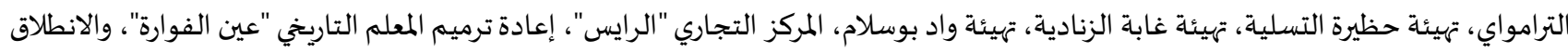
في الكشف عن حفريات "المدينة الرومانية" التي ظهرت بملعب "قصياب"، بالإضافة إلى إنشاء العديد من الفنادق المصنفة التي دخلت حيز الخدماة...،

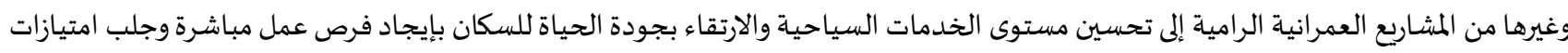
تنموية باعتبارها مصدر دائم للتنمية الحضرية، تعمل على الرفع من حجم التدفق السياحي الوافد للمدينة وزيادة حجم الاستثمار السياحي.

2.1 - 2.1 أهداف الدراسة:

ترمي الدراسة إلى:

توضيح دور المشاريع العمرانية المجسدة بمدينة سطيف كفرص لتعزيز تنمية السياحة الحضرية فيها. تحديد مختلف التحولات الحضرية الحاصلة بعد تنفيذ مختلف المشاريع العمرانية والتي سمحت كلها بتوسع فرص السياحة الحضرية من خلال تزايد مجالات الراحة والاستجمام وقضياء أوقات الفراغ...

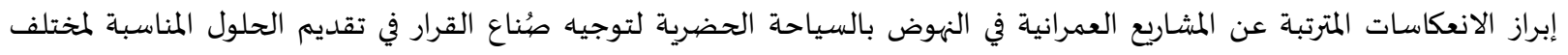

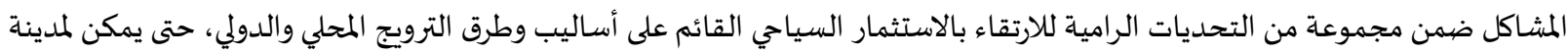
سطيف أن تحتل مكانة هامة في سوق السياحة الدولي كمدينة كبرى بمنطقة الهضياب العُليا. 
3.1. - 3.1 أدبيات الدراسـة:

لم تلقى السياحة الحضرية اهتمامًا واضحًا من قبل الباحثين إلا مع بداية سنة 1970 بالمملكة المتحدة وأوروبا الغربية وأمريكا الشمالية، نتيجة

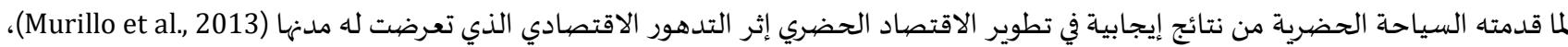

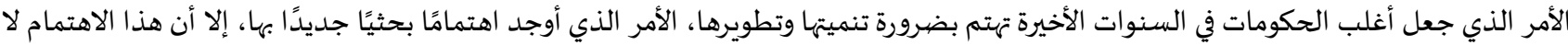

يعكس مدى الأهمية التي تكتسبها السياحة في أنواع أخرى من الوجهات. (Estelaji et al., 2012) ومع بداية عشرية الثمانيات استفادت العديد من مدن العالم من مشـاريع عمرانية تمثلت في تجديد حضري للمناطق التراثية التاريخية والذي

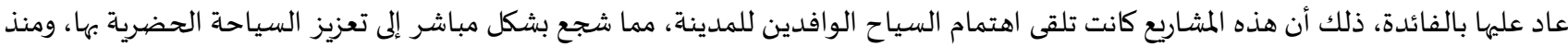
هذه الفترة أصبحت السياحة الحضرية محل اهتمام مختلف الباحثين والدارسين (Van Den Berg et al., 1995)، والذين عملوا بشكل كبير على لهئ إعطاء مفاهيم للسياحة الحضرية، وتحديد الفرص والتحديات التي تواجهها، ولعل أهم هذه الاهتمامات نذكر أعمال Dharmwani سنة اهنة 2013 الذي الذي

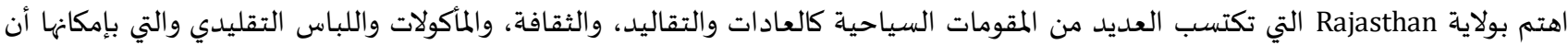

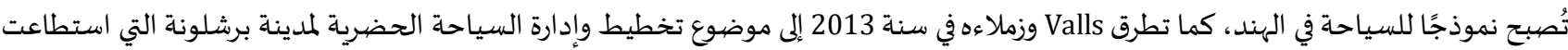

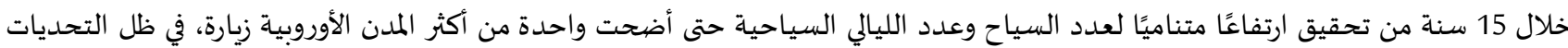

المتعلقة بالقدرة التنافسية للمدينة خاصة مع غياب توازن مكاني في توزيع الوجهات السياحية بالمدينة. (Valls et al., 2013) وفي سنة 2015 ظهرت أبحاث Kidane-Mariam التي تناولت موضوع تنمية السياحة الحضرية بدولة أثيوبيا بالتركيز على العاصيمة أديس أبابا،

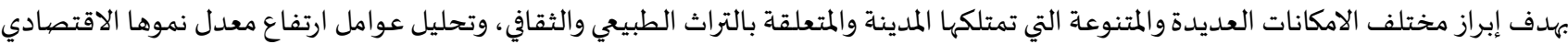

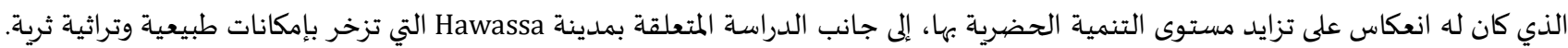

(Kidane-Mariam, 2015)

أما في سنة 2017، فقد تطرق Shiji إلى توضيح مفهوم السياحة الحضرية والعوامل المؤثرة فيها، وعناصرها المختلفة (الأسـاسية، والثانوية

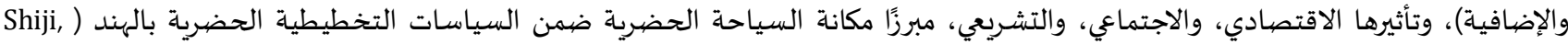
2017)، وفي نفس السنة، تناول Ababneh,Al-Saad في بحثيهما تشخيص الوضع الحالي للسياحة العربية مستندين في ذلك إلى ثلاثة مدن عربية، وهي:

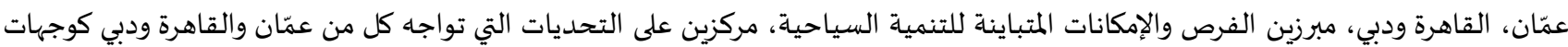
رئيسية (Al-Saad, Ababneh, 2017)

ومع تنامي أهمية السياحة الحضرية على الصعيد العالمي ومساهمتها في تنمية المجتمع اقتصاديًا، واجتماعيًا وثقافيًا وبيئيًا، والاعتراف بها دوليًا كأهم الوظائف الرئيسة بالمدن والتي تعرف تطورًا مستمرًا (Hakeem, Khan, 2018)، استمر الاهتمام بموضيوع السياحة الحضرية في العديد من الدراسات والبحوث التي عملت على ضرورة وجود تسويق سياحي للوجهات السياحية الحضرية من أجل تطويرها، وهو ما يتضح فئس في دراسة Khreis و سنة 2019 في دراسة مقارنة بين مدينتي قسنطينة (الجزائر) وعمّان (الأردن) والتي أوضحت وجود فوارق كبيرة في إستراتيجية

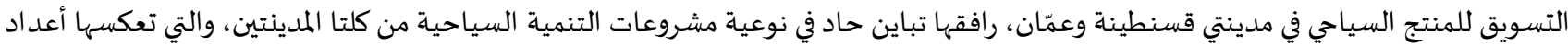

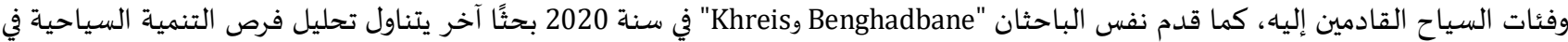
أربعة مدن عربية ساحلية، وهي العقبة (الأردن) والإسكندرية (مصر) بمنطقة الشرق الأوسط، وعنابة الهية (الجزائر) والدار البيضاء (المغرب) بشمال أفريقيا، والمرتبطة بخصوصية موقعها الجغرافي الساحلي في ممارسة العديد من الأنشطة السياحية بها (الحضرية، الثقافية، الشاطئية، الدينية...)، وهذه المدن تعاني من مشاكل متعدّدة مرتبطة بالتصنيع وما ترتب عناه من ظهور للتلوث بأشكال مختلفة، إلى جانب موسمية السوق السياحية وانتشار

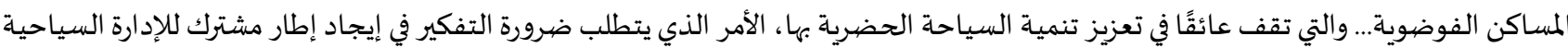

الرشيدة والمتكاملة بالمدن الساحلية العربية. (Benghadbane, Khreis, 2020)

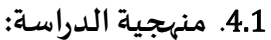

من أجل تشخيص مختلف الفرص التي توفرها مدينة سطيف لتعزيز السياحة الحضرية بها، فإن الدراسة اعتمدت على المنهج الوصفي لتوضيح

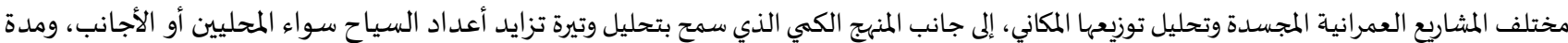
إقامتهم، مع إبراز مختلف الانعكاسـات الاقتصادية والاجتماعية والعمرانية لهذه المشاريع العمرانية التي على ضوءها يمكن توجياه صُّناع القرار للنهوض

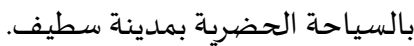
كما استندت هذه الدراسة على مجموعة من المراجع النظرية كالكتب والبحوث والدراسات لجمع البيانات والمعلومات المرتبطة بموضوع البحث وكذا مجال

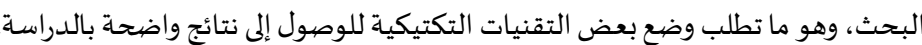


2. تحليل ومناقشة:

1.2. موقع جغرافي يدعم السياحة الحضرية:

يلعب الموقع الجغرافي للمدينة دورًا كبيرًا في خلق فرص الجذاِب السياحي وتطويره، وتنويعه بالمجال الحضري، وهو ما نجده قائمًا في مدينة

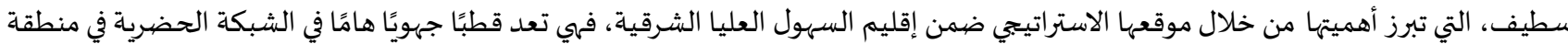

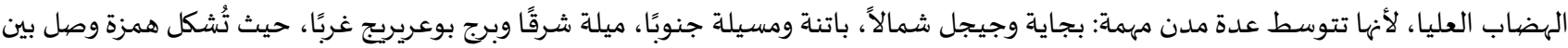

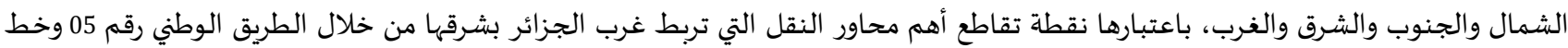
السكة الحديدية والطريق السيار "شرق-غرب"، وشمالها بجنوبها عبر الطرق الوطنية ذات الأرقام: 28،09،78 (خريطة: 1)، مما يؤهلها لتكون عاصيمة

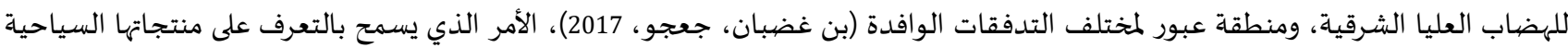
من قبل مختلف الوافدين إلهها من جهات متعددة. إلى جانب قربها من مختلف المحطات المعدنية المهمة التي تعد بمثابة مناطق لقيام السياحة العلاجية

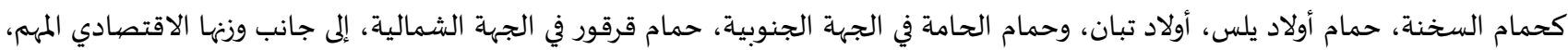
وتعدد مشاريع التهيئة الحضرية بها وقربها من المواقع السياحية (المدينة الرومانية "جميلة"، المنطقة الأثرية "واد الصفصاف"، آثار الدولة الفاطمية ببني

عزيز)

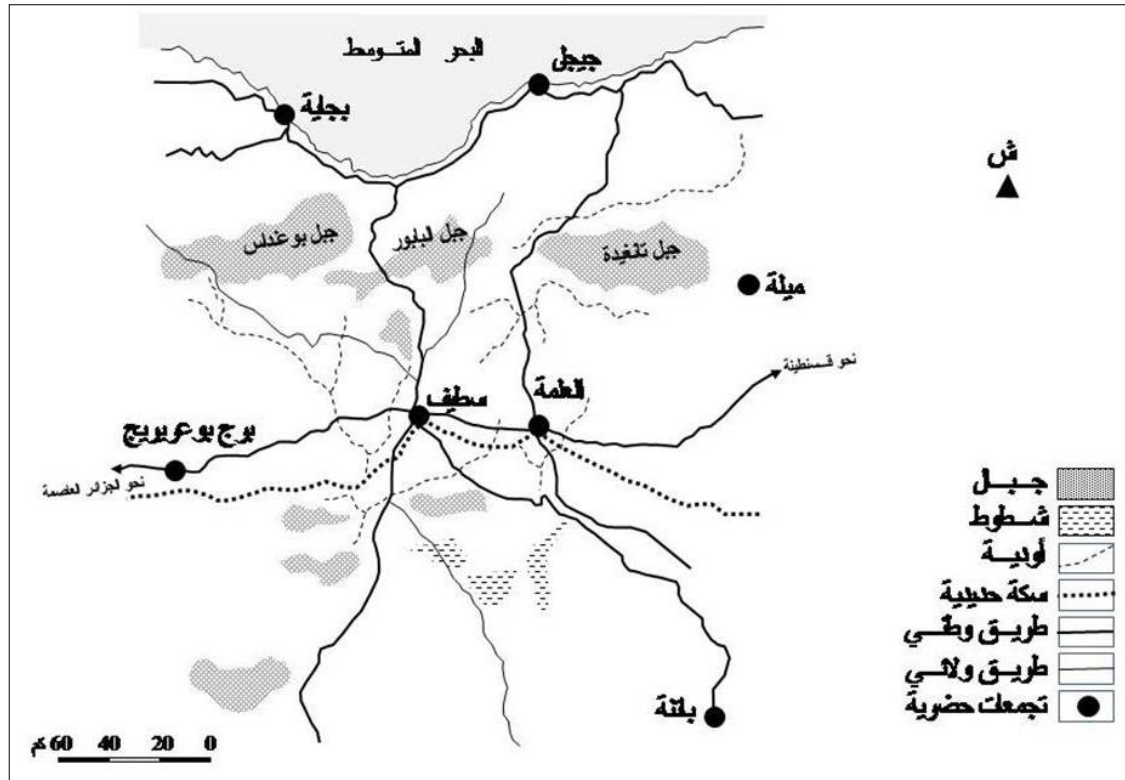

خربطة (1): الموقع الجغرافي لمدينة سطيف

المصدر: عمل الباحثين انطلاقًا من الخربطة الطبوغرافية، سطيف (1/50.000).

فرص متعددة للسياحة الحضرية:

تتوزع أهم فرص السياحة الحضرية على مستويين مكانيين هامين، أولهما مستوى المجال الحضري لمدينة سطيف، والثاني على على المستوى الولائي. فمدينة سطيف تتوفر على عدد من المعالم والمواقع السياحية المنتشرة عبر نسيجها العمراني، والتي تستقطب العديد من الوافدين إلها بهدف

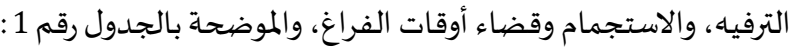

جدول (1): توزيع المعالم والمو اقع السياحية بمدينة سطيف

\begin{tabular}{|c|c|}
\hline الخصيائص & المعلم والموقع \\
\hline 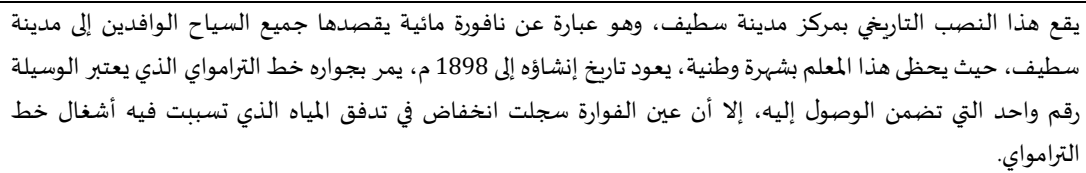 & المعلم التاريخي "عين الفوارة" \\
\hline 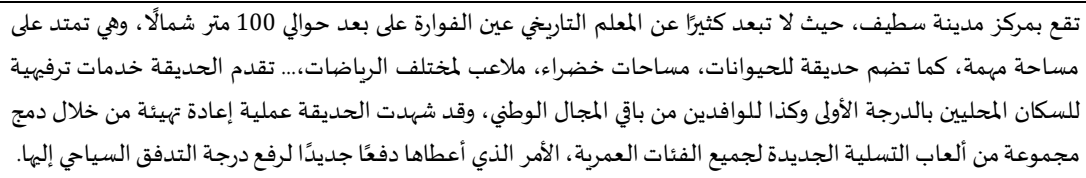 & حديقة التسلية "8 ماي 1945" \\
\hline
\end{tabular}




\begin{tabular}{|c|c|}
\hline 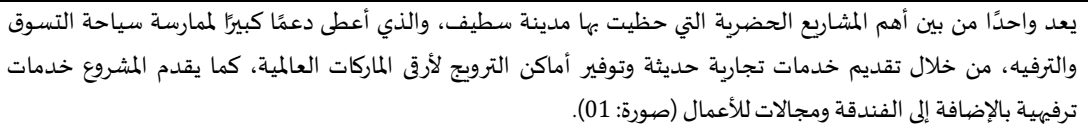 & المركز التجاري "العالي" \\
\hline 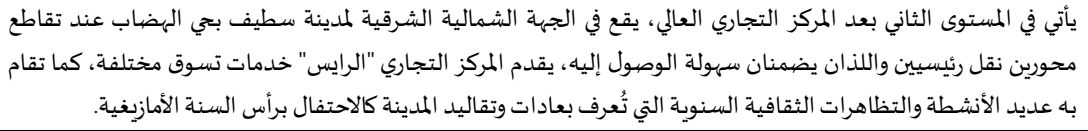 & المركز التجاري "الر ايس" \\
\hline والسياحي لاستقبال السياح الوافدين إلهيها. إلفترة البيزنية تشاؤه سنة 540 م، شهدت القلعة عملية إعادة ترميم لإعادة إدماجها ضمن النشاط & القلعة البيزنطية \\
\hline والفوارة" ، حيث يعكس ذاكرة تاريخية للمدينة المساجد العتيقة بمدينة سطيف علعود تاريخ إنشاءه إلى العهد العثماني، يقع بمحاذاة النصب التاريخي "عين & مسجد العتيق \\
\hline 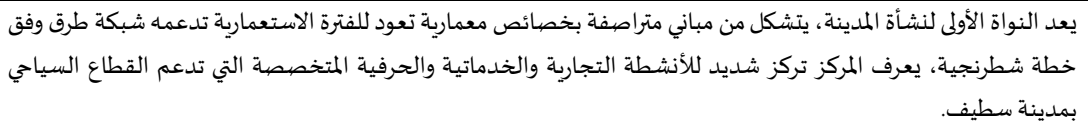 & المركز الأوروبي \\
\hline يعجد المتحف من الوطني فضاء سياحي بمدينة سطيف، يعطي صورة مفصلة عن مختلف الحضارات المتعاقبة على المدينة من خلال & المتحف الوطني \\
\hline 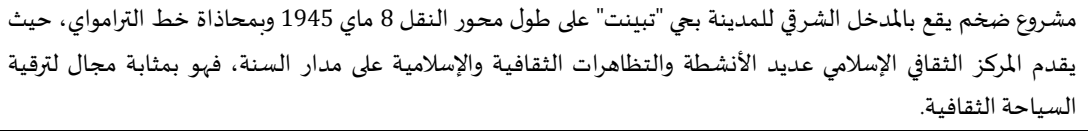 & المركز الثقافي الإسلامي \\
\hline 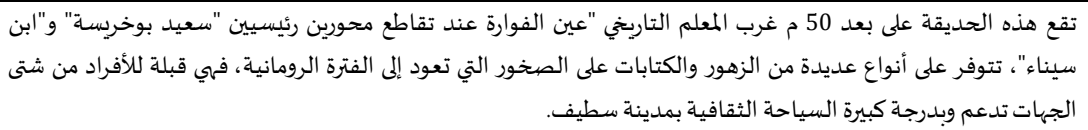 & الحديقة الأثرية "الأمير عبد القادر" \\
\hline للتوقف، أضاف سكني تلمدينة يقع في المدخل الشرقي للمدينة يقداري معاصر من خلال خصوصية واجدماته متنوعة سكعمارية، تجارية، ترفيهية...، كما يوفر فضاء كبير & المشروع السكني التج \\
\hline
\end{tabular}

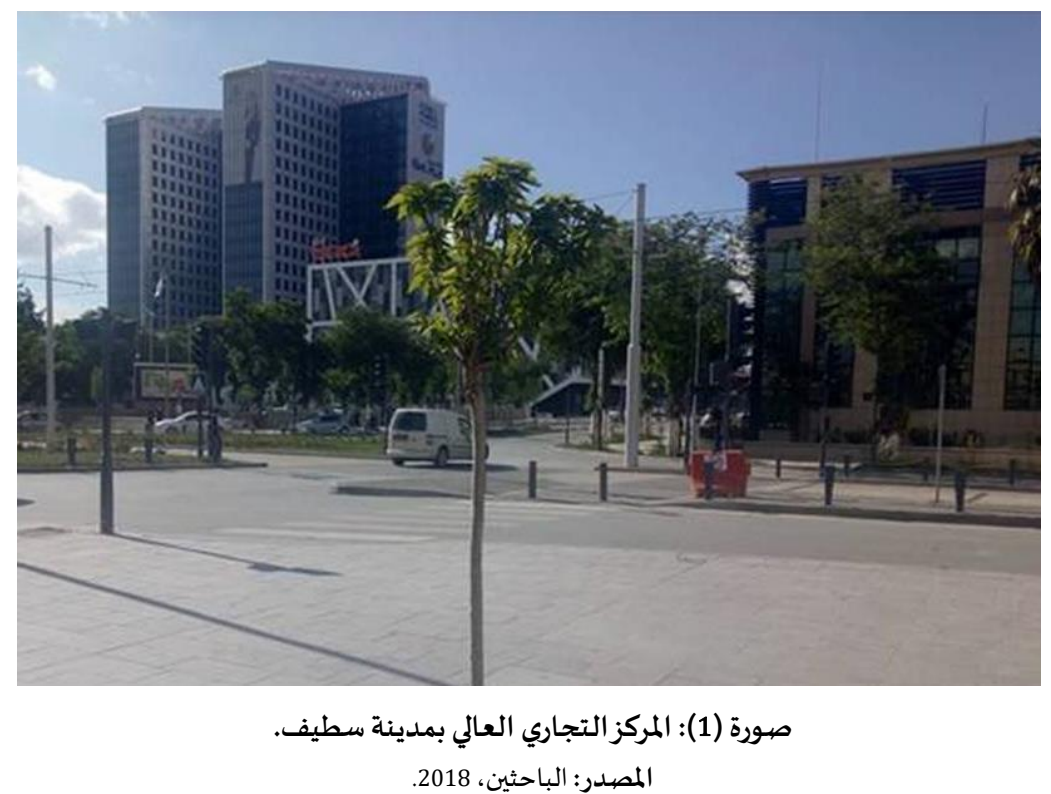

والجدير بالذكر، أن هناك العديد من المشاريع الحضرية التي لم يتم ذكرها، ونخص هنا مشروع تهيئة "غابة الزنادية" بالجهة الشمالية لمدينة

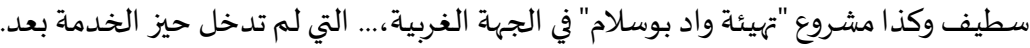

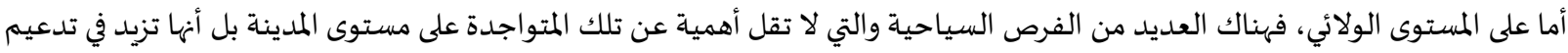

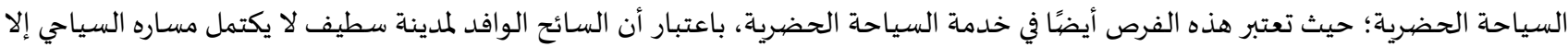
بزيارة هذه الأماكن الموضحة بالجدول رقم 2 : 


\begin{tabular}{|c|c|}
\hline الخصائص & المعلم والموقع \\
\hline 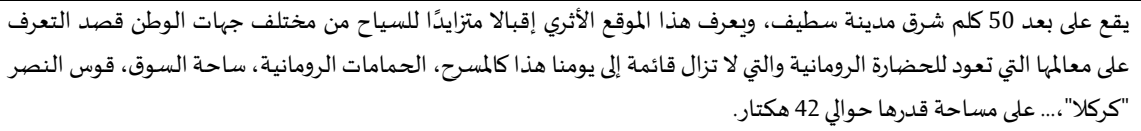 & الموقع الأثري "جميلة" \\
\hline 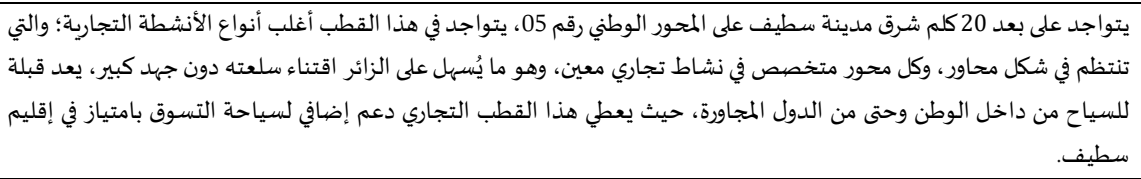 & القطب التجاري "دبي" بالعلمة \\
\hline 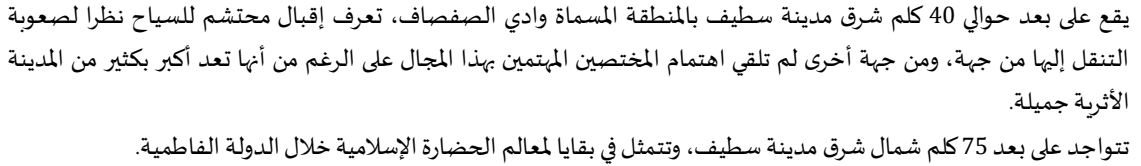 & 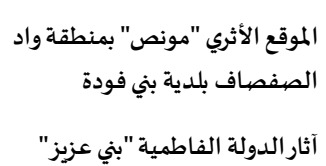 \\
\hline يعود هذا الموقع إلى عصور ما قبل التاريخ بمنطقة "القلتة الزرقاء" على بعد 35 كلم شرق مدينة سطيف، تم اكتشافه من قبل & 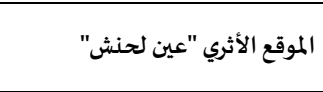 \\
\hline 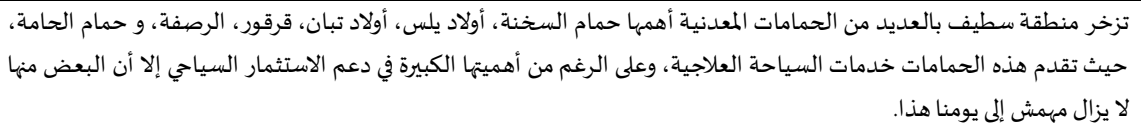 & الحمامات المعدنية \\
\hline 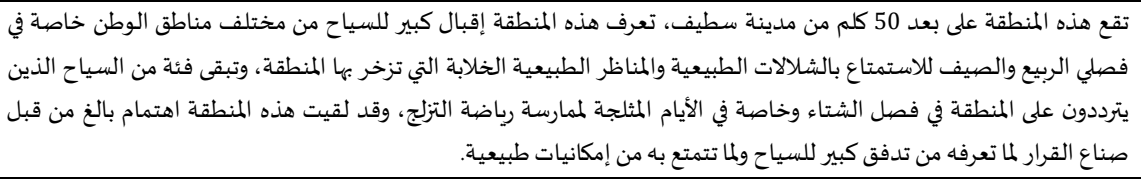 & منطقة السياحة الطبيعية "بابور" \\
\hline
\end{tabular}

3.2. تحولات حضبرية ودورمتز ايد للمشاريع العمر انية في تنشيط السياحة الحضرية:

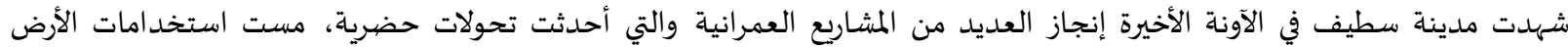

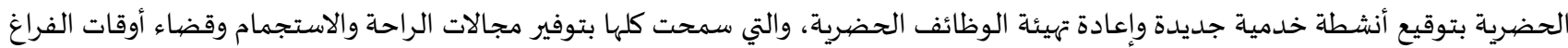
لسكان المدينة وحتى للوافدين إليها، وهذا ما سيكون له انعكاسات واضحة لتفعيل السياحة الحضرية. وتعتبر المشاريع المرتبطة بالإيواء من أهم المشاريع العمرانية، وتتمثل في عدد الفنادق المنجزة في مدينة سطيف لتوسيع طاقة الاستيعاب السياحي، والتي زاد عددها من 40 فندق سنة 2016 إلى 45 فندق سنة 2018 من أصل 72 فندق منها ثلاثة سلاسل فندقياة: FOUR POINTS BY ACCORHOTELS (IBIS \& NOVOTEL) - BEST WESTERN -SHERATON
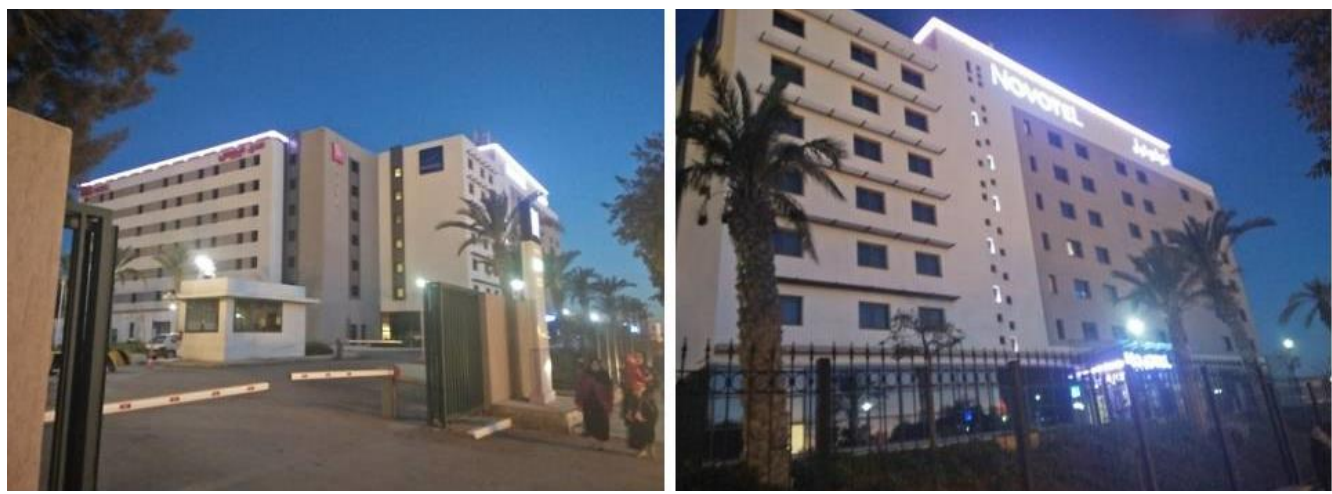

صورة (2): بعض فنادق مدينة سطيف

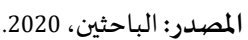

كما تُسجل المشاريع المرتبطة بالنقل أهميتها الكبيرة في تطوير السياحة الحضرية، لما لها من دور في الربط بين مختلف المواقع السياحية وتسهيل

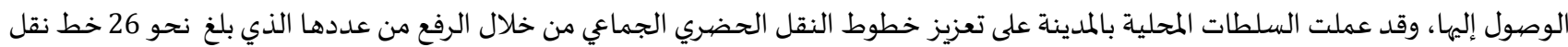
حضري خاص و5 خطوط نقل حضري عمومي (خريطة: 2) تغطي احتياجات التنقل داخل المدينة وفي كل الاتجاهات بسعة إجمالية قدرت بـ 457 حافلة

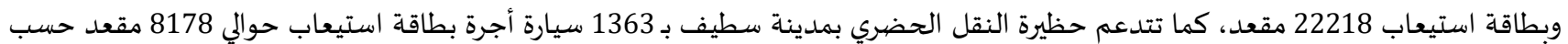
الإحصائيات المنجزة من طرف مديرية البرمجة ومتابعة الميزانية لولاية سطيف لسنة 2015. 


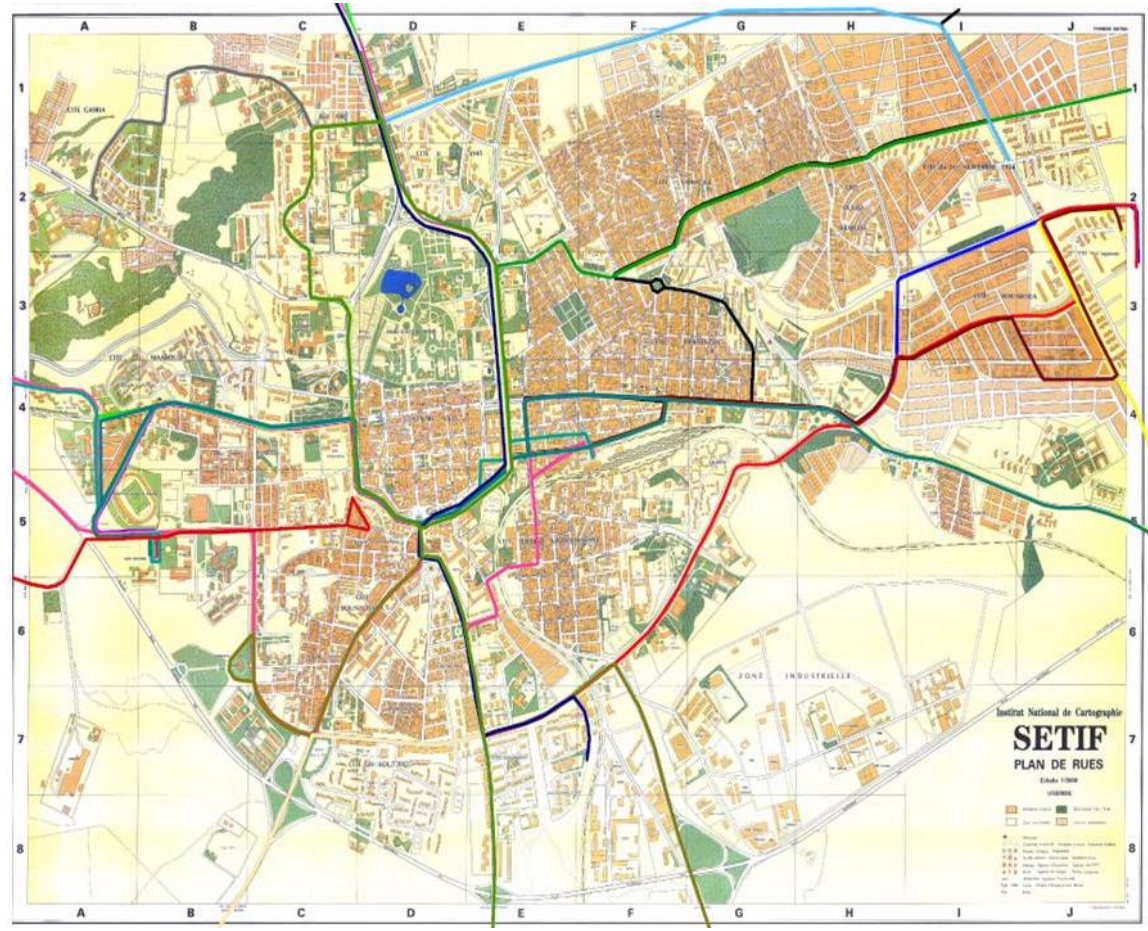

خريطة (2): مسارات النقل الحضري بمدينة سطيف

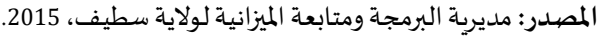

إلى جانب إنجاز مشروع "خط الترامواي" الذي يُعد مبادرة جد مهمة في توفير النقل الآمن والبيئي، يتشكل المشروع في الأساس من ثلاثة خطوط

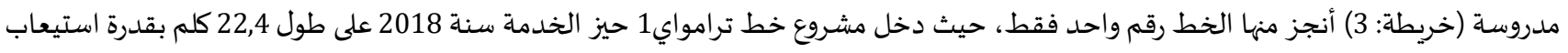
تقدر بـ 5000 راكب/ساعة في الإتجاه الواحد مرورًا بـ 22 محطة، منها خمس محطات مهمة مرتبطة مباشرة بالمعالم التاريخية والمشاريع العمرانياة السياحية والمتمثلة في محطة "محمد بوضياف" المتواجدة بالقرب من المركز الثقافي الإسلامي، ومحطة "مصطفى بن بوالعيد" المجاورة للمتحف الوطني

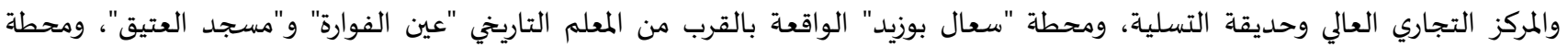

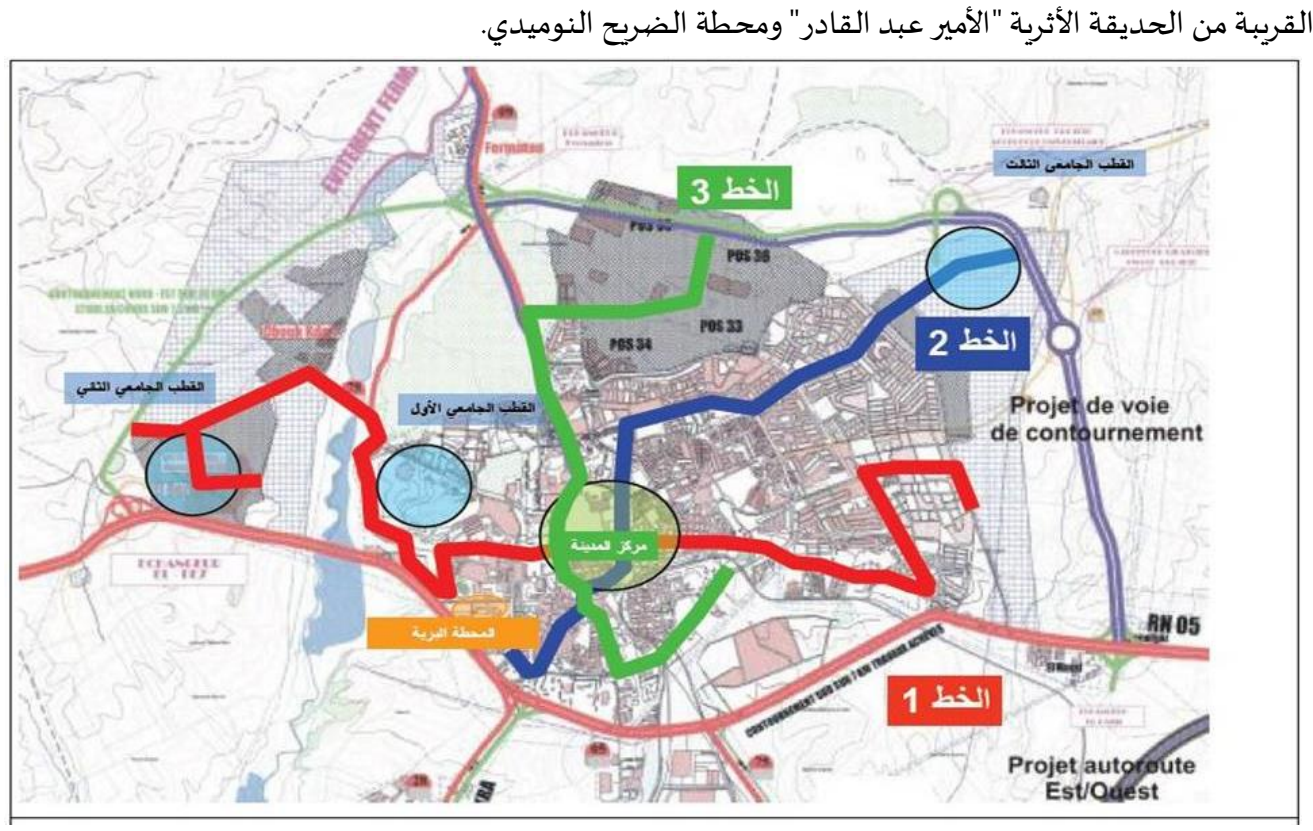

خريطة (3): خطوط النقل بالتوامواي في مدينة سطيف المبرديف

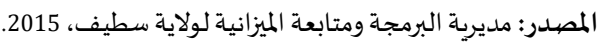


كما تم إنجاز مشـاريع أخرى مرتبطة بجميع الخدمات التي تدعم القطاع السياحي بمدينة سطيف والمتمثلة في البنوك والمصيارف المالية التي تعمل على تنشيط التنمية السياحية، وقد بلغ عددها حوالي 15 بنك تتوطن معظمها بمركز المدينة ، أهمها: ABC BANK. BDL. TRUST BANK. ARAB

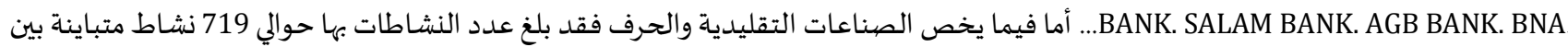
صناعات تقليدية فنية، وصناعات تقليدية لإنتاج المواد، وكذا الصناعات التقليدية لإنتاج الخدمات، إذ بلغ عدد الحرفيين 16303 حرفي يشغلون

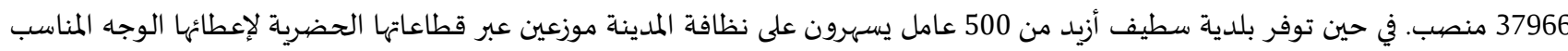

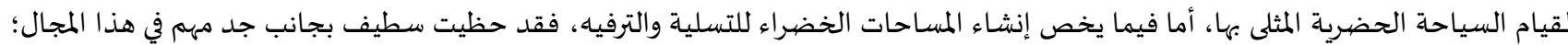

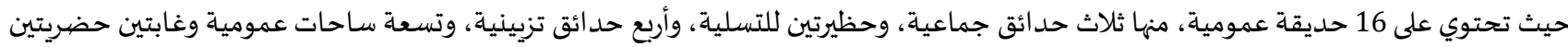
بمساحة إجمالية تُقدر بنحو 76.31 هكتار (وداعي، بويبية، 2015)، ضف إلى ذلك سلسلة المطاعم الراقية أهمها مطعم "اليمامة" المتواجد بالمدخل الشرقي للمدينة، ومطعم "مشاوي حلب"، ومطعم "دار الضياف" في الجهة الغربية للمدينة...، وغيرها.

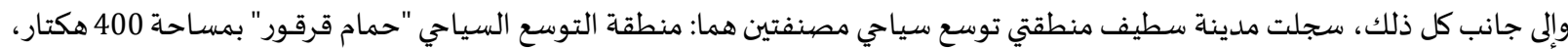
ومنطقة التوسع السياحي "مقرس" بمساحة 215.50 هكتار، وثلاث مناطق سياحية غير مصنفة، والمتمثلة في: منطقة التوسع السياحي "حمام السخنة" بمساحة تقدر بـ 133 هكتار، منطقة التوسع السياحي حمام سيدي عمر بلدية الحامة بمساحة تقدر بـ بماحس 40 هكتار، ومنطقة التوسع السياحي حمام

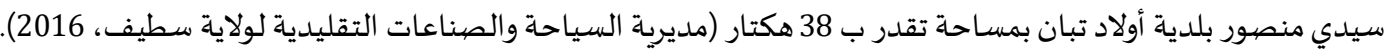

\section{3.}

1.3. المشاريع العمر انية ساعدت على تعزيز السياحة الحضرية: لعبت مشاريع التخطيط الحضري التي استفادت منها مدينة سطيف عبر مراحل زمنية مختلفة دورا كبيرا في إحداث ديناميكيكية هيكية هامة على

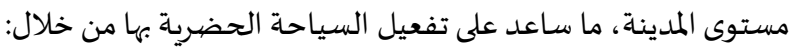
تجسيد مشاريع الربط والاتصال بين أحياء المدينة، خاصة من خلال مشروع الترامواي الرابط بين الجهة الغربية والشرقية للمدينة، والذي أحدث

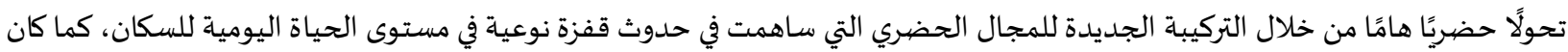
للمشروع خطوة كبيرة في دعم السياحة الحضرية من خلال ربط مختلف المواقع والمعالم التاريخية المتواجدة بالمدينة والتي يسهل على السائح التنقل إليها في أحسن الظروف. تحسين المظهر الحضري من خلال مختلف برامج التحسين الحضري التي استفادت منها مختلف أحياء مدينة سطيف كحي 600 مسكن، حي

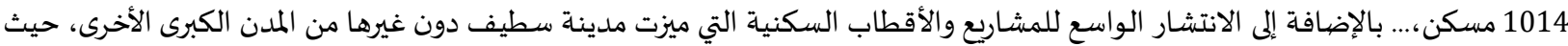
احتضنت قيام أول صالون للترقية العقارية والتجهيز والديكور بمدينة سطيف سنة 2018 بمشاركة أزيد من 20 مؤسسة عقارية. العمليات الدورية لأعوان النظافة التابعين لمؤسسات خاصة" "ECOSET" المتخصصة بجمع، ونقل، ومعالجة النفايات عبر مختلف القطاعات

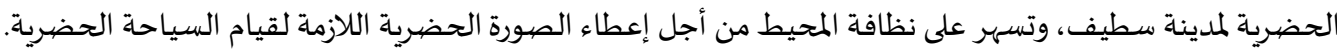
تدعيم الاقتصاد الحضري وتفعيل حركيته من خلال الرسوم التي توفرها المشاريع العمرانية في مجال الخدمات السياحية (مواقف سيارات، المراحيض العمومياة، حظائر التسلية، مطاعم، مقاهي،...) وغيرها من المشاريع التي يستغلها السياح أثناء تنقلاتهم اليومية داخل مدينة سطيف. زيادة التوافد السياحي على الفنادق المنجزة بمدينة سطيف، والذي تطور عددهم من 40 فندق سنة 2016 بسعة استيعاب قدرت بحوالي 2594

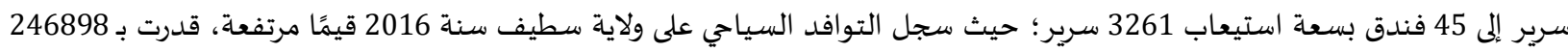

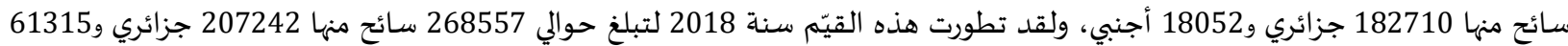

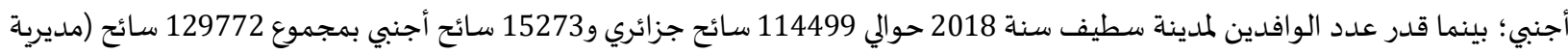
السياحة والصناعات التقليدية لولاية سطيف، 2019)، والتي ستساهم دون شك في زيادة الديناميكية الحضرية التي تدعم بشكل مباشر

الاقتصاد الحضري.

مشاكل تعيق تنمية السياحة الحضرية:

بالرغم من أهمية المشاريع العمرانية المجسدة في مدينة سطيف والدور الطوعي البارز من قبل صناع القرار للنهوض بالسياحة الحضرية بمدينة

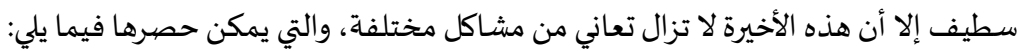
تأخر انطلاق العديد من مشاريع الاستثمار السياحي والمقدرة بـ 22 مشروع، نتيجة عدم الحصول على رخص البناء وأخرى تتعلق بمشاكل مرتبطة بالمستثمر في حد ذاته، وكذا 7 مشاريع استثمار سياحي متوفقة في انتظار تسوية نزاعات قضيائية. غياب الترويج السياحي للصورة الذهنية لمدينة سطيف وخاصة على المستوى الدولي القادر على الرفع من حجم التدفق السياحي. 
غياب شبه كلي لمشاركة المجتمع المحلي في استراتيجية التنمية الحضرية بمدينة سطيف. غياب خريطة سياحية لمدينة سطيف، توضح أهم الفرص السياحية من معالم ومواقع تاريخية وسياحية بالمدينة. هشاكل متعلقة بجودة الخدمات السياحية والتي تتضح من خلال التدني في مستواها (نقل، إيواء..). ضعف في عمليات إعادة الترميم للمواقع الأثرية وعدم تصنيفها يجعلها عرضاة للتخريب بشتى أنواعاه. ا الاستعمال الضعيف والنادر لأساليب التكنولوجيا والذكاء في قطاع السياحة بمدينة سطيف يحول دون النهوض بالسياحة الحضرية وتطويرها. غياب الأمن بشتى أنواعها في العديد من المواقع السياحية. رغم تعدّد مشاريع التهيئة الحضرية المنجزة بمدينة سطيف والتي من شأنها تدعم دفع عجلة التنمية السياحية إلا أها غير كافية لاستقطاب السياح الأجانب. وجود العديد من المشاكل المرتبطة بالتنقل على مستوى المدينة وخاصةة في أوقات الذروة، مما ينعكس على راحة السائح لمدينة سطيف. مشاكل متعلقة بالتوقف وخاصة في الأماكن التي تتواجد بها المعالم التاريخية كعين الفوارة، وكذا أماكن الترفيه والتسوق كالمركز التجاري العالي وحظيرة التسلية.

3.3. تصورات مستقبلية لتعزيز السياحة الحضرية بمدينة سطيف: على غرار المشاكل المطروحة التي يعرفها قطاع السياحة بمدينة سطيف يمكن تقديم جملة من التوصيات التي من شأهها تفعيل السياحة الحضرية بها في ظل التحديات التي تواجهها مدينة سطيف، وذلك من خلال: ضبط مبادئ وتوجهات مخطط التهيئة السياحية لآفاق 2030، والسهر على تجسيده على أرض الواقع للنهوض بالسياحة في مدينة سطيف. ضرورة حل المشاكل والنزاعات المتعلقة بالمشاريع الاستثمار السياحي سواء المتوقفة أو التي لم تنطلق بعد. إعادة النظر في المشاريع العمرانية الكلاسيكية وضرورة تبني سياسـة جديدة تقوم على توطين مشاريع بمجال تأثير يتعدى حدود إقليم الولاية الكفيل بتحقيق الجاذبية السياحية لمدينة سطيف.

تكوين صورة سياحية ذهنية لمدينة سطيف قادرة على الترويج للفرص السياحية التي تزخر بها المدينة. ضرورة مشاركة المجتمع المحلي في كل المشاريع العمرانية لتدعيم السياحة الحضرية، وذلك لدوره الكبير في الترويج للمنتوج الثقافي للمدينة من خلال الارتقاء بالصناعات والحرف التقليدية المحلية. العمل على الارتقاء بالمواقع التراثية الثرية المنتشرة بكثافة بمدينة سطيف. الحرص على تنويع الأنشطة السياحية باختلاف أنواعها بمدينة سطيف التي تتوفر فيها كل الفرص السياحية. وضع خريطة واضحة المعالم توزع بالمناطق التي تعرف توافد كبير للسياح قصد استغلالها لزيارة مناطق أخرى يجهلونها. إدخال عنصر الذكاء في تقديم الخدمات السياحية بجميع أنواعها كطلب سيارة أجرة، حجز غرفة بفندق معين،... وغيرها. السهر على أمن وسلامة السائح سواء الأجنبي أو الوطني. حل جميع المشاكل المتعلقة بالتنقل والتوقف والتشجيع على استعمال وسائل النقل الجماعي للتقليل من عدد السيارة الفردية. وضع خطوط نقل تجوب المدينة مرورا بمعالمها التاريخية. الأخذ بتجارب السياحة الحضرية الناجحة للمدن السباقة، وتكييف إيجابياتها مع خصيوصية مدينة سطيف.

خلاصية:

يتضح من كل ما سبق تعدّد فرص السياحة الحضرية بمدينة سطيف والتي تدعمت من خلال الدور الكبير لعمليات التخطيط الحضري التي ساهمت بشكل كبير في توفير مناطق الترفياه والاستجمام وقضاء أوقات الفراغ، إلى جانب توسيع الخدمات السياحية لاسيما من ناحية النقل والإيواء،

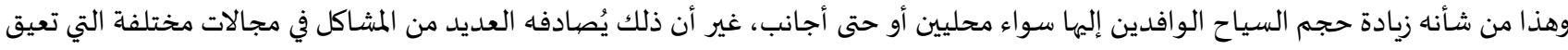
تطوير السياحة الحضرية بالمدينة والتي تتطلب ضرورة مشاركة بين جميع الفاعلين مع صناع القرار بهدف التنسيق بين المشاريع العمرانية وإستراتيجية

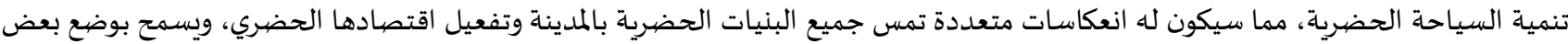

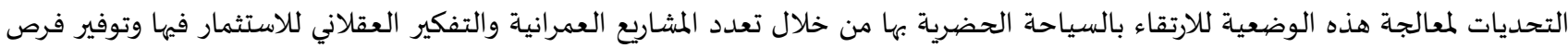
الشغل بالاعتمادها على أساليب وطرق ترويجية متجددة باستمرار على مستويات متعددة داخلية ودولية، تعمل على جلب أكبر عدد من السياح ومن 
فئات محددة ومقصودة للرفع من إيراداتها، مدعمة بجملة من التحفيزات، لاحتلال مكانة متميزة بالسوق السياحي الدولي والذي يتدعم من خلال موقعها الجغرافي بالهضياب العليا.

1. سليماني، نبيل (2009). دراسة تصهميم وتنفيذ مشروع نظام معلومات جغرافي SIG لتسيير المجال: حالة ولاية سطيف. مذكرة ماجستير في التهيئة

الإقليمية. جامعة قسنطينة: الجزائر.

2. صالجي، عاطف (2005). وضع خُطة تنموية ضهمن أبعادها البيئية بولاية سطيف. مذكرة ماجستير في التهيئة العمرانياة. جامعة قسنطينة: الجزائر.

$$
\text { 3. بن غضبان، فؤاد (2015). السياحة البيئية المستدامة: بين النظرية والتطبيق. دار صفاء للنشر والتوزيع. عمّان: الأردن. }
$$

5. بن غضبان، فؤاد، جعجو، محفوظ (2018).ترقية المنتجات السياحية وتنويعها كبدائل استراتيجية لتحقيق التنمية السياحة المتوازنة بالجزائر:

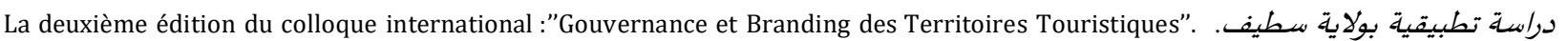
Université Internationale d'Agadir-Universiapolis : Maroc. 26-27-28 mars.

6 6. بن غضبان، فؤاد، جعجو، محفوظ (2017). التوجه المكاني في تقييم جودة الحياة الحضرية بملدينة سطيف (الجزائر). مجلة العمارة والتخطيط. مجلد: 29 (1): 63-87.

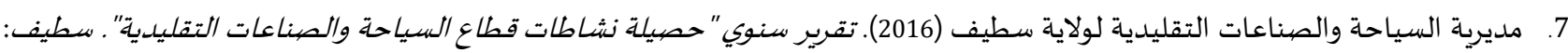

8. مديرية السياحة والصناعات التقليدية لولاية سطيف (2019). تقرير سنوي. سطيف: الجزائر.

9 9. وداعي منير، بويبية عادل (2015). التوسع العمداني في إطار التنمية المستد/مة: ملدينة سطيف. منكرة ماستر في تسيير الملدن والتنمية المستدامة.

$$
\text { معهد تسيير التقنيات الحضرية. جامعة أم البواقي: الجزائر. }
$$

ثانياً: المراجع الأجنبية:

1. Al-Saad, S. \& Ababneh, A.K. (2017). Concept, opportunities and challenges of urban tourism in the Arab world: Case studies of Dubai, Cairo and Amman. Tourism Review: An International Interdisciplinary Journal. 65(3): 361-375.

2. Blaževic, B. \& Krstinic Nižic, M. (2015). City organization as urban challenge for tourism destinations. Tourism in Southern and Eastern Europe. 3rd International Scientific Conference Tourism in Southern and Eastern Europe. Tourism in Southern and Eastern Europe, 3: 19-33.

3. Benghadbane, F. \& Khreis, S. (2019). The role of tourism marketing in enhancing tourism development: a comparative study between Constantine and Amman cities. GeoJournal of Tourism and Geosites, 24(1):146-160, https://doi.org/10.30892/gtg.24112-349.

4. Benghadbane, F. \& Khries, S. (2020). Urban touristic development in the coastal cities: case study: Aqaba, Alexandria, Annaba and Casablanca cities. GeoJournal of Tourism and Geosites, 29(2): 488-507, https://doi.org/10.30892/gtg.29209-484.

5. BET GCNERU. (2013). Enjeux de l'aménagement et du développement touristique de la wilaya de SétifES6/DES//02/11/CA, Phase 2, Sétif, Algérie.

6. Bock, K. (2015). The changing nature of city tourism and its possible implications for the future of cities. European Journal of Futures Research, 3(1):1-8, https://doi.org/10.1007/s40309-015-0078-5.

7. Bouadam K. (2011). The national strategy of tourism development in Algeria: issues, opportunities and limitations. Review of Applied Socio-Economic Research, 2: 1-15.

8. Direction du Tourisme et de l'Artisanat de la Wilaya de Sétif. (2012). Le rapport d'établissement : le lancement de l'étude du SDAT -SETIF, SDATW -SETIF-PH1, Sétif, Algérie.

9. Estelaji, A., Sadeghian, H. \& Beyhaghi, Z. (2012). The role of urban tourism to achieve urban sustainable development. International Journal of Geology, 6(1): 17- 25

10. Hayllar, B., Griffin, T. \& Edwards, D. (2008). City Spaces - Tourist Places: Urban Tourism Precincts. ButterworthHeinemann is an imprint of Elsevier: Oxford- UK, Burlington: USA. 
11. Hakeem, A. \& Khan, H. (2018). Urban Tourism: The Perspective on Tourism Impacts in Cambridge, United Kingdom. Marketing and Management of Innovations, 24(3): 268- 275, https://doi.org/10.21272/mmi.2018.3-24.

12. Kidane-Mariam, T. (2015). Ethiopia: Opportunities and Challenges of Tourism Development in the Addis Ababa-upper Rift Valley Corridor. Journal of Tourism and Hospitality, 4(4): 1- 9, https://doi.org/10.4172/2167-0269.1000167.

13. Murillo, J., Vayá, E., Romaní, J. \& Surinach, J. (2013). How important to a city are tourists and day-trippers? The economic impact of tourism on the city of Barcelona. Tourism Economics, 19(4): 897-917, https://doi.org/10.5367/te.2013.0225.

14. Nogués-Pedregal, A.M. (2019). The instrumental time of memory: local politics and urban aesthetics in a tourism context. Journal of Tourism Analysis. https://doi.org/10.1108/JTA-05-2018-0014.

15. Shiji, O. (2017). Urban tourism: The case of India. International Journal of Advanced Education and Research, 2(6): 30-36.

16. Valls, J.F., Banchini, S., Falcón \& L.Tuñon, G.V. (2013). Repositioning of Barcelona's Image in the Light of a Redefinition of the Urban Tourism Planning Model. Revista de Turismo y Patrimonio Cultural, 11(1): 89-105, https://doi.org/10.25145/j.pasos.2013.11.005.

17. Yildiz, S. \& Akbulut, M.T. (2013). Current trends in developing urban tourism. International Journal of Architectural Research, 7(2) : 297-310. 
International Journal of Hospitality and Tourism Studies (IJHTS)

Journal Homepage: https://www.refaad.com/views/IJHTS/home.aspx

www.refaad.com

ISSN 2709-0892 (Online) ISSN 2709-0884 (Print)

\section{The role of urban projects in the enhancement of urban tourism in Algerian cities: Opportunities, transformations, and challenges Case: the city of Setif}

\section{Foued Benghadbane}

Laboratory for the evaluation of the quality of uses in architecture and in the built environment LEQUAE Institute of Management of the Urban Techniques, University of Oum El Bouaghi, Algeria foued.benghadbane@univ-oeb.dz

\section{Djaadjou Mahfoud}

Department of Architecture, University of Chlef, Algeria mahfouddj89@gmail.com

Received: 1/5/2021 Revised: 18/5/2021 Accepted: 21/6/2021 DOI: https://doi.org/10.31559/IJHTS2020.2.1.1

Abstract: Abstract: The concept of tourism is no longer limited to travel with the aim of seeing other places distinguished by different cultures and customs only. Rather, it has expanded to make tourism more practiced in cities within a new concept, which is "urban tourism", which represents the approach taken by most governments in strengthening their cities to be distinguished in finding urban tourism projects, with new urban and architectural designs, through which they reflect images of tourism investments in cities.

In this context, we will highlight the city of Setif, considered one of the largest cities of Algeria in the eastern highlands, in order to clarify the various major urban and functional transformations resulting from the implementation of numerous urban projects (hotel chain, large shopping centers, tramway project, etc.), as well as to reorganize various urban functions within it, this has made it possible to provide many leisure spaces and its layout, to attract many tourists coming to the city, in particular with ease of movement towards it. This has led the city of Setif to offer many opportunities which are necessary to develop its urban tourism.

We will try to highlight the different urban mutations that have affected the city of Setif in various economic, social and urban areas, to discover all the problems that hamper the development of urban tourism in the city, based on descriptive approaches and quantitative and certain tactical techniques, as well as all the references and resources available on the theme of research and its spatial field, on the basis of which certain recommendations likely to pose challenges can be visualized, thanks to it, a strategy global can be developed to improve the city so that it becomes a destination for urban tourism locally and internationally.

Keywords : urban tourism, opportunities, transformations, leisure spaces, challenges, Sétif, Algeria.

\section{References:}

1. Bn Ghdban, F'ad (2015). Alsyahh Alby'yh Almstdamh: Byn Alnzryh Walttbyq. Dar Sfa' Llnshr Waltwzy'. 'man: Alardn.

2. Bn Ghdban, F'ad (2019). Altkhtyt Alsyahy Mn Ajl Tnmyt Syahyh Mstdamh. Dar Sfa' Llnshr Waltwzy'. 'man: Alardn.

3. Bn Ghdban, F'ad, J'jw, Mhfwz (2017). Altwjh Almkany Fy Tqyym Jwdt Alhyah Alhdryh Bmdynh Styf (Aljza'r). Mjlt Al'marh Waltkhtyt. Mjld: 29 (1): 63-87.

4. Bn Ghdban, F'ad, J'jw, Mhfwz (2018). Trqyh Almntjat Alsyahyh Wtnwy'ha Kbda'l Astratyjyh Lthqyq Altnmyh Alsyahh Almtwaznh Baljza'r: Drash Ttbyqyh Bwlayh Styf. La Deuxième Édition Du Colloque International:"Gouvernance Et Branding Des Territoires Touristiques". Université Internationale d'Agadir-Universiapolis : Maroc. 26-27-28 Mars. 
5. Mdyryt Alsyahh Walsna'eat Altqlydyh Lwlayh Styf (2016). Tqryr Snwy "Hsylt Nshatat Qta' Alsyahh Walsna'at Altqlydyh". Styf: Aljza'r.

6. Mdyryt Alsyahh Walsna'at Altqlydyh Lwlayh Styf (2019). Tqryr Snwy. Styf: Aljza'r.

7. Salhy, 'atf (2005). Wd' Khuth Tnmwyh Dmn Ab'adha Alby'yh Bwlayh Styf. Mdkrt Majstyr Fy Althy'h Al'mranyh. Jam't Qsntynh: Aljza'r.

8. Slymany, Nbyl (2009). Drash Tsmym Wtnfyd Mshrw' Nzam M'lwmat Jghrafy SIG Ltsyyr Almjal: Halh Wlayh Styf. Mdkrt Majstyr Fy Althy'h Aleqlymyh. Jam't Qsntynh: Aljza'r.

9. Wda'y Mnyr, Bwybyh 'adl (2015). Altws' Al'mrany Fy Etar Altnmyh Almstdamh: Mdynt Styf. Mdkrt Mastr Fy Tsyyr Almdn Waltnmyh Almstdamh. M'hd Tsyyr Altqnyat Alhdryh. Jam't Am Albwaqy: Aljza'r. 